\title{
Another approach to non-repetitive colorings of graphs of bounded degree
}

\author{
Matthieu Rosenfeld* \\ Aix Marseille Université, Université de Toulon, CNRS, LIS, \\ Marseille, France \\ matthieu.rosenfeld@gmail.com
}

Submitted: Jun 23, 2020; Accepted: Aug 3, 2020; Published: Sep 4, 2020

(C) The author. Released under the CC BY-ND license (International 4.0).

\begin{abstract}
We propose a new proof technique that applies to the same problems as the Lovász Local Lemma or the entropy-compression method. We present this approach in the context of non-repetitive colorings and we use it to improve upper-bounds relating different non-repetitive chromatic numbers to the maximal degree of a graph. It seems that there should be other interesting applications of the presented approach.

In terms of upper-bounds our approach seems to be as strong as entropycompression, but the proofs are more elementary and shorter. The applications we provide in this paper are upper bounds for graphs of maximal degree at most $\Delta$ : a minor improvement on the upper-bound of the non-repetitive chromatic number, a $4.25 \Delta+o(\Delta)$ upper-bound on the weak total non-repetitive chromatic number, and a $\Delta^{2}+\frac{3}{2^{1 / 3}} \Delta^{5 / 3}+o\left(\Delta^{5 / 3}\right)$ upper-bound on the total non-repetitive chromatic number of graphs. This last result implies the same upper-bound for the non-repetitive chromatic index of graphs, which improves the best known bound.
\end{abstract}

Mathematics Subject Classifications: 05C15

\section{Introduction}

A sequence $s_{1} \ldots s_{2 n}$ is a square if $s_{i}=s_{i+n}$ for each $i \in\{1, \ldots, n\}$. A sequence is repetitive if it contains a consecutive subsequence that is a square and it is non-repetitive (or square-free) otherwise. For instance, the words hotshots, repetitive and alfalfa are repetitive and the words total and minimize are square-free.

The work of Thue on non-repetitive words is regarded as the starting point of combinatorics on words $[18,19]$ (see [4] for a translation in modern mathematical English). He

\footnotetext{
*The author was partially supported by the ANR project CoCoGro (ANR-16-CE40-0005)
} 
showed that there are infinite square-free sequences over three elements. Many generalizations and variations of this notion have been studied. One such notion that received a lot of attention is the notion of non-repetitive coloring of graphs introduced by Alon et al. [1] (see [6, 20] for surveys on this topic). We say that a coloring (either of the vertices or of the edges) of a graph is non-repetitive if the sequence of colors of any path is nonrepetitive. The non-repetitive chromatic number (resp. non-repetitive chromatic index) (also called Thue number and Thue index) of a graph, denoted by $\pi(G)$ (resp. $\pi^{\prime}(G)$ ) is the smallest number of colors in a non-repetitive coloring of the vertices (resp. the edges) of the graph. Alon et al. showed that $\pi^{\prime}(G)$ is in $O\left(\Delta^{2}\right)$ where $\Delta$ is the maximum degree of $G$ [1]. Different authors successively improved the upper-bounds on the non-repetitive chromatic number and the non-repetitive chromatic index $[13,8,10]$. Although this is not the topic of this article, non-repetitive colorings have since been studied in many other context than graphs of bounded degree. For instance, after many intermediate results it was recently shown that planar graphs have bounded non-repetitive chromatic number [7].

Most results regarding non-repetitive coloring of graphs of bounded maximal degree are either based on the Lovász Local Lemma $(L L L)$ or entropy-compression and they naturally hold in the stronger setting of list coloring. A list assignment of a graph $G$ is a function that maps any vertex $x$ (and/or any edge) to a set of colors $L(x)$. An $L$-coloring of a graph is a coloring of the graph such that the color of each vertex (and/or edges) $x$ belongs to its list of colors $L(x)$. A graph is then said to be non-repetitively $\ell$-choosable if it admits a non-repetitive $L$-coloring for any list assignment $L$ such that every list is of size at least $\ell$. The non-repetitive choice number $\pi_{\mathrm{ch}}(G)$ is the smallest integer $\ell$ such that $G$ is non-repetitively $\ell$-choosable. Similarly, the list variant of the non-repetitive chromatic index is denoted by $\pi_{\mathrm{ch}}^{\prime}(G)$. The best bounds relating these quantities to the maximum degree $\Delta$ of a graph are respectively (see [10])

$$
\pi_{\mathrm{ch}}^{\prime}(G) \leqslant \Delta^{2}+2^{4 / 3} \Delta^{5 / 3}+O\left(\Delta^{4 / 3}\right)
$$

and

$$
\pi_{\mathrm{ch}}(G) \leqslant\left\lceil\Delta^{2}+\frac{3}{2^{2 / 3}} \Delta^{5 / 3}+\frac{2^{2 / 3} \Delta^{5 / 3}}{\Delta^{1 / 3}-2^{1 / 3}}\right\rceil .
$$

The notion of Total Thue coloring was introduced by Schreyer and Škrabuvláková [17]. A coloring of the edges and the vertices of a graph is a weak total Thue coloring if the sequence of consecutive vertex-colors and edge-colors of every path is non repetitive. If moreover the sequence of vertex-colors and the sequence of edge-colors of any path are both non-repetitive then this is a (strong) total Thue coloring. The weak total Thue number $\pi_{\mathrm{Tw}}(G)$ (resp. total Thue number $\pi_{\mathrm{T}}(G)$ ) is the minimum number of colors in a weak total Thue coloring of $G$ (resp. a total Thue coloring of $G$ ). These two parameters both have there list-coloring counterpart denoted respectively by $\pi_{\mathrm{Twch}}(G)$ and $\pi_{\mathrm{Tch}}(G)$. Schreyer and Škrabuvláková [17] showed that $\pi_{\mathrm{T}}(G) \leqslant 5 \Delta^{2}+o\left(\Delta^{2}\right), \pi_{\mathrm{Tch}}(G) \leqslant 17.9856 \Delta^{2}$ and $\pi_{\mathrm{Tw}}(G) \leqslant|E(G)|-|V(G)|+5$. We remark that the second bound also relies on an application of the Lovász Local Lemma.

In this article, we propose a different proof technique strongly related to the Lovász Local Lemma and to entropy-compression (whose idea is based on the algorithmic proof 
of the Lovász Local Lemma by Moser and Tardos [14]) and we apply this technique to different non-repetitive coloring problems. This technique provides bounds as good as entropy-compression, but is much more elementary. The more advanced mathematics required in the proof is summation of geometric series (for comparison, entropy-compression arguments usually rely on analytic combinatorics to compute some variations of Catalan numbers to bound the number of records). The main idea of this approach is to show inductively that, if we color the vertices or edges one-by-one, at every "step" the number of possible colorings grows exponentially (this resembles the proof of LLL in this regard). One more benefit of this approach is that it provides exponential lower bounds on the number of solutions. However, we lose the constructive aspect and the algorithmic implications of entropy-compression arguments. We should mention that Bernshteyn recently introduced the Local Cut Lemma [3], a lemma that also aims to be applied to the same set of problems as LLL or entropy-compression. In some cases it provides shorter proofs than LLL or entropy-compression, but they are still more technical (and it might be argued that they are longer if one includes the proof of the Local Cut Lemma itself).

As a simple illustration of our technique we first provide a proof that the Thue choice number of any path is at most 4 (for a proof of this result based on LLL see [12] and for a proof based on entropy-compression see [11]). Then we apply our technique to the Thue choice number in Theorem 3 and improve the lowest degree term of the bound given by (2). The improvement is minor and could certainly be achieved with a more careful analysis in [10], however the proof given in [10] is much more technical than our self-contained proof of one and a half pages.

We then apply our method to weak total Thue coloring and total Thue coloring. Our first result given in Theorem 5 asserts that $\pi_{\text {Twch }}(G) \leqslant 6 \Delta$ for any graph of maximum degree $\Delta$. In Theorem 7 , we show that if $\Delta \geqslant 300$, we have a stronger bound $\pi_{\text {Twch }}(G) \leqslant$ 4.25 $\Delta$. Our result is not obvious to compare to the bound $\pi_{T w}(G) \leqslant|E(G)|-|V(G)|+5$ from [17], but it is stronger in most graphs (also our result is more general since for any graph $\left.G, \pi_{\mathrm{Tw}}(G) \leqslant \pi_{\mathrm{Twch}}(G)\right)$. For instance, as long as the average degree of the graph is at least 9.5 our bound is stronger. In fact, our result also implies a linear upper-bound on the number of vertices instead of a quadratic upper-bound.

Regarding the total Thue choice number, we show in Theorem 9 that $\pi_{T}(G) \leqslant$ $\pi_{\mathrm{Tch}}(G) \leqslant \Delta^{2}+\frac{3}{2^{1 / 3}} \Delta^{5 / 3}+8 \Delta^{4 / 3}+1$ which improves considerably the two bounds from [17] previously mentioned and slightly improves the bound for the non-repetitive chromatic index. We conclude with a discussion regarding applications of this method to other problems.

We will use the term " $L$-coloring" for partial colorings if all the colored elements receive a color from their list and if the context clearly indicates that the coloring is partial. Recall that for any $x>1$,

$$
\sum_{i \geqslant 1} i x^{-i}=\frac{x}{(x-1)^{2}}
$$




\section{Non-repetitive colorings of paths and the proof technique}

In this section, we first give an illustrative example of our proof technique. Then we informally sketch a more general description of the proof technique.

\subsection{Non-repetitive colorings of paths}

This section is devoted to the proof of Theorem 1 . This result is not new $[12,11]$, but it is a simple application of our approach.

Theorem 1. For every path $P, \pi_{\mathrm{ch}}(P)=4$.

This Theorem is a simple consequence of the following lemma. We order the vertices of any path "from left to right" such that each vertex is adjacent to the vertex to its right and to its left (the leftmost and rightmost vertices are the ends of the path).

Lemma 2. Let $L$ be a list assignment of a path $P$ such that all lists are of size 4 . Let $C_{n}$ be the number of non-repetitive $L$-colorings of the $n$ leftmost vertices of $P$. Then for any integer $n<|P|$,

$$
C_{n+1} \geqslant 2 C_{n}
$$

Proof. Let us proceed by induction on $n$. Let $n$ be an integer such that the Lemma holds for any integer smaller than $n$. We now show that $C_{n+1} \geqslant 2 C_{n}$. Let $F$ be the set of $L$-colorings of the $n+1$ leftmost vertices that are repetitive, but whose restrictions to the $n$ leftmost vertices are non-repetitive. Then

$$
C_{n+1}=4 C_{n}-|F|
$$

Let us now bound the size of $F$. For every $i$, let $F_{i}$ be the colorings from $F$ that contain a square of length $2 i$. Then clearly $|F| \leqslant \sum_{i \geqslant 1}\left|F_{i}\right|$. For any coloring $c$ of $F_{i}$, the first $n+1-i$ colors form a non-repetitive coloring and they uniquely determine the last $i$ colors of $c$ because of the repetition. We deduce that for all $i,\left|F_{i}\right| \leqslant C_{n+1-i}$. By the induction hypothesis this implies that $\left|F_{i}\right| \leqslant 2^{1-i} C_{n}$. We finally get

$$
C_{n+1} \geqslant 4 C_{n}-\sum_{i \geqslant 1} 2^{1-i} C_{n} \geqslant 2 C_{n}
$$

which concludes our proof.

The consequences of Lemma 2 are in fact stronger than Theorem 1, since it implies that there are at least $2^{n}$ colorings for any list assignment. However, the statement of this Lemma is even stronger and it is crucial in the proof that the number of valid colorings is multiplied by at least 2 every time that we color one more vertex. 


\subsection{The proof technique}

Let us now give a more general informal sketch of our proof technique. Suppose one wants to show that any graph from some class $\mathcal{C}$ of graphs admits a valid coloring with at most $\gamma$ colors (eg. Theorem 1). Suppose moreover that both the class and the valid colorings are hereditary in the sense that the graph induced by a colored elements also belongs to $\mathcal{C}$ and that every subcoloring of a valid coloring is also valid. For any graph $G$, we let $c(G)$ be the set of valid colorings of $G$.

We try to show the stronger result that there exists a constant $\alpha>1$ such that for any graph $G \in \mathcal{C}$ and element $e$ of $G$ to be colored (it might be an edge or a vertex), $|c(G)| \geqslant \alpha \times|c(G-e)|$ (eg, Lemma 2 with $\gamma=4$ and $\alpha=2)$. We proceed by induction on $|V(G)|$ to show this result.

Let $F$ be the set of colorings of $G$ that are not valid, but whose restrictions to $G-e$ are valid colorings. Then by definition,

$$
|c(G)|=\gamma|c(G-e)|-F .
$$

Now suppose we can find coefficients $\left(a_{i}\right)_{i \geqslant 1}$ such that

$$
|F| \leqslant \sum_{i \geqslant 1} a_{i} \frac{|c(G-e)|}{\alpha^{i-1}}
$$

and

$$
\sum_{i \geqslant 1} \frac{a_{i}}{\alpha^{i-1}} \leqslant(\gamma-\alpha)
$$

We deduce

$$
|c(G)| \geqslant|c(G-e)| \alpha
$$

which concludes our proof.

The technical part is to show the upper-bound on $F$ with the right coefficients $\left(a_{i}\right)_{i \geqslant 1}$. This is done by finding injections from $F$ to valid colorings of subgraphs of $G-e$. More precisely, we find a way to express $F$ as the union of colorings $\left(F_{i}\right)_{i \geqslant 1}$, such that for all $i$ there is an injection from $F_{i}$ to the union of the colorings of $a_{i}$ different subgraphs of $G-e$ of cardinality $|G|-i$. Then we can use our induction hypothesis to upper-bound the number of valid colorings of any subgraph obtained by removing $i$ elements of $G-e$ by $\frac{|c(G-e)|}{\alpha^{i}}$ which leads to equation (3).

It might seem that we have to guess the right values of $\gamma$ and $\alpha$ in the Lemma statement, but it is not the case. Indeed, one can first find the coefficients $\left(a_{i}\right)_{i \geqslant 1}$ with variables $\gamma$ and $\alpha$ and then take the best values $\gamma$ and $\alpha$ such that equation (4) is satisfied. This is done by choosing

$$
\gamma=\min _{\alpha>\frac{1}{r}}\left(\alpha+\sum_{i \geqslant 1} \frac{a_{i}}{\alpha^{i-1}}\right)
$$


where $r$ is the radius of convergence of $\sum_{i \geqslant 1} a_{i} x^{i} .{ }^{1}$ For instance, in Lemma 2, we need $\gamma-\frac{\alpha}{\alpha-1} \geqslant \alpha$ and $\alpha>1$. The minimum of $\frac{\alpha}{\alpha-1}+\alpha$ is 4 and is reached for $\alpha=2$ these are respectively the best values to take for $\gamma$ and $\alpha$.

The technique is strongly related to the entropy-compression technique. In fact, in the particular context of colorings of graphs of bounded degree, it is equivalent to the approach of [10, Theorem 12]. Using our technique one can in fact provide a simpler proof of their Theorem 12 (our $a_{i}$ are their $C_{i}$ and our $\alpha$ is their $x^{-1}$ ), but it does not seem to be worth the trouble of introducing all the necessary formalism only to provide an alternative proof of the exact same result. However, even if we can simplify the proof and match the bound of their Theorem, we cannot easily improve the bound.

Remark that bounding $|F|$ in the proof of Lemma 2 is simplified by the linear structure of the path, since we know that the vertices that contribute to a square are always the last vertices added. In fact, in the setting of words this proof is almost identical to the power series method for pattern avoidance $[2,5,15,16]$.

\section{Non-repetitive colorings}

In this section we apply our method to non-repetitive colorings of graphs of bounded maximum degree.

Theorem 3. For every graph $G$ with maximum degree $\Delta \geqslant 1$,

$$
\pi_{\mathrm{ch}}(G) \leqslant \Delta^{2}+\frac{3}{2^{2 / 3}} \Delta^{5 / 3}+2^{2 / 3} \Delta^{4 / 3} .
$$

Let us instead show a stronger Lemma. For any graph $G$ and any list assignment $L$ of $G$, the set $C_{L}(G)$ is the set of non-repetitive $L$-colorings of $G$.

Lemma 4. Let $\Delta \geqslant 2$ be an integer and $\gamma=\frac{3}{2^{2 / 3}}+2^{2 / 3} \Delta^{-1 / 3}+\Delta^{-2 / 3}$. Let $G$ be a graph of maximal degree at most $\Delta$ and $L$ be a list assignment of $G$. If each list is of size at least $\Delta(\Delta-1)\left(1+\gamma \Delta^{-1 / 3}\right)+1$, then for any vertex $v$ of $G$,

$$
\left|C_{L}(G)\right| \geqslant \Delta(\Delta-1)\left(1+2^{1 / 3} \Delta^{-1 / 3}\right)\left|C_{L}(G-v)\right| .
$$

Proof. Let us show this by induction on the number of vertices of $G$. This is clearly true if $|G|=1$ since the empty graph has exactly one coloring. Let $n$ be an integer such that the Lemma holds for any graph with less than $n$ vertices.

Let $G$ be a graph over $n$ vertices of maximal degree at most $\Delta$ and $L$ be a list assignment of $G$ such that each list is of size at least $\Delta(\Delta-1)\left(1+\gamma \Delta^{-1 / 3}\right)+1$. Let $v$ be any vertices of $G$.

Let $F$ be the set of repetitive $L$-colorings of $G$ whose restriction to $G-v$ is a nonrepetitive coloring. Then

$$
\left|C_{L}(G)\right|=\left(\Delta(\Delta-1)\left(1+\gamma \Delta^{-1 / 3}\right)+1\right)\left|C_{L}(G-v)\right|-|F| .
$$

\footnotetext{
${ }^{1}$ In pratice, we take upper-bounds of the minimum obtained from manipulations of Taylor polynomials in order to give nicer expressions.
} 
We need to upper-bound the size of $F$. Let $F_{i}$ be the set of colorings from $F$ that contain a path of length $2 i$ that is a square. Clearly $|F| \leqslant \sum_{i \geqslant 1}\left|F_{i}\right|$. Thus for any coloring $c$ from $F_{i}$ there is path $p$ of length $2 i$ such that

- the sequence of colors of $p$ is a square,

- $p$ contains $v$ and let us call $p^{\prime}$ the half of $p$ that contains $v$ and $G^{\prime}$ the graph obtained by removing from $G$ the vertices of $p^{\prime}$ and the adjacent edges,

- the restriction of $c$ to $G^{\prime}$ is non-repetitive,

- $p$ and the restriction of $c$ to $G^{\prime}$ uniquely determines $c$ (since the second half of the square is identical to the first half).

Thus, for any fixed $p$ and $p^{\prime}$, the number of such colorings from $F_{i}$ is at most $\left|C_{L}\left(G^{\prime}\right)\right|$. Since $p^{\prime}$ contains $v$ and $i-1$ other vertices our induction hypothesis implies that this quantity is bounded by

$$
\left|C_{L}\left(G^{\prime}\right)\right| \leqslant \frac{\left|C_{L}(G-v)\right|}{\left(\Delta(\Delta-1)\left(1+2^{1 / 3} \Delta^{-1 / 3}\right)\right)^{i-1}} .
$$

Moreover, there are at most $i \Delta(\Delta-1)^{2 i-2}$ paths of length $2 i$ going through $v$. To see that, remark that $v$ splits such a path in two halves, so one can choose the length of the shortest half between 0 and $i-1$. Then there are $\Delta$ ways to chose the neigbor of $v$ that belongs to the long half of the path and after that there are $\Delta-1$ possible choices for each new vertex to add to the path. We deduce

$$
\left|F_{i}\right| \leqslant i \Delta(\Delta-1)^{2 i-2} \frac{\left|C_{L}(G-v)\right|}{\left(\Delta(\Delta-1)\left(1+2^{1 / 3} \Delta^{-1 / 3}\right)\right)^{i-1}} .
$$

We simplify the computations with the slightly weaker bound for $i \geqslant 2$,

$$
\left|F_{i}\right| \leqslant i(\Delta-1) \frac{\left|C_{L}(G-v)\right|}{\left(1+2^{1 / 3} \Delta^{-1 / 3}\right)^{i-1}}
$$

and for $i=1$ we use

$$
\left|F_{1}\right| \leqslant \Delta\left|C_{L}(G-v)\right|=(\Delta-1)\left|C_{L}(G-v)\right|+\left|C_{L}(G-v)\right| .
$$

We finally upper-bound $|F|$.

$$
\begin{aligned}
|F| & \leqslant\left|C_{L}(G-v)\right|\left(1+(\Delta-1) \sum_{i \geqslant 1} \frac{i}{\left(1+2^{1 / 3} \Delta^{-1 / 3}\right)^{i-1}}\right) \\
& \leqslant\left|C_{L}(G-v)\right|\left(1+(\Delta-1) \frac{\left(1+2^{1 / 3} \Delta^{-1 / 3}\right)^{2}}{\left(\left(1+2^{1 / 3} \Delta^{-1 / 3}\right)-1\right)^{2}}\right) \\
& \leqslant\left|C_{L}(G-v)\right|\left(1+(\Delta-1) \Delta^{2 / 3}\left(2^{-1 / 3}+\Delta^{-1 / 3}\right)^{2}\right)
\end{aligned}
$$


Together with equation (5), it implies

$$
\begin{aligned}
\left|C_{L}(G)\right| & \geqslant\left|C_{L}(G-v)\right|\left(\Delta(\Delta-1)\left(1+\gamma \Delta^{-1 / 3}\right)-(\Delta-1) \Delta^{2 / 3}\left(2^{-1 / 3}+\Delta^{-1 / 3}\right)^{2}\right) \\
& \geqslant\left|C_{L}(G-v)\right| \Delta(\Delta-1)\left(1+\Delta^{-1 / 3}\left(\gamma-\left(2^{-1 / 3}+\Delta^{-1 / 3}\right)^{2}\right)\right) \\
& \geqslant\left|C_{L}(G-v)\right| \Delta(\Delta-1)\left(1+\Delta^{-1 / 3}\left(\gamma-2^{-2 / 3}-2^{2 / 3} \Delta^{-1 / 3}-\Delta^{-2 / 3}\right)\right) .
\end{aligned}
$$

Substituting $\gamma=\frac{3}{2^{2 / 3}}+2^{2 / 3} \Delta^{-1 / 3}+\Delta^{-2 / 3}$, we finally get

$$
\left|C_{L}(G)\right| \geqslant\left|C_{L}(G-v)\right| \Delta(\Delta-1)\left(1+\Delta^{-1 / 3} 2^{1 / 3}\right)
$$

which concludes this proof.

Remark that the bound given by this Lemma is in fact

$$
\begin{aligned}
\pi_{\mathrm{ch}} & \leqslant\left\lceil\Delta(\Delta-1)\left(1+\frac{3}{2^{2 / 3}} \Delta^{-1 / 3}+2^{2 / 3} \Delta^{-2 / 3}+\Delta^{-1}\right)+1\right\rceil \\
& \leqslant\left\lceil\Delta^{2}+\frac{3}{2^{2 / 3}} \Delta^{5 / 3}+2^{2 / 3} \Delta^{4 / 3}-\frac{3}{2^{2 / 3}} \Delta^{2 / 3}-2^{2 / 3} \Delta^{1 / 3}\right\rceil
\end{aligned}
$$

which is slightly stronger than the bound given in Theorem 3. One can also compare the bound from Theorem 3 to the result from [10] (already mentioned in equation (2)). As $\Delta$ goes to $+\infty$ their upper-bound is asymptotically equivalent to

$$
\Delta^{2}+\frac{3}{2^{2 / 3}} \Delta^{5 / 3}+2^{2 / 3} \Delta^{4 / 3}+2 \Delta+\mathcal{O}\left(\Delta^{2 / 3}\right)
$$

which is larger than our upper-bound by $2 \Delta+\mathcal{O}\left(\Delta^{2 / 3}\right)$. This is a really minor improvement and this can be achieved with the entropy-compression argument. The best known lowedbound on the maximal Thue number for any maximum degree $\Delta$ is $\Omega\left(\frac{\Delta^{2}}{\log \Delta}\right)$ [1], so this could be the case that even the first coefficient is not optimal. However, the fact that our method is simpler allowed us to easily improve the analysis while still providing a shorter proof.

\section{4 (Weak) total Thue coloring}

In this section, we consider three kinds of paths

- vertex-paths: sequences of consecutive adjacent vertices (they were simply called path in the previous section),

- edges-paths: sequences of consecutive adjacent edges,

- mixed-paths: alternating sequences of vertices and edges such that consecutive elements are adjacent. 
In each of these definitions, we require that there are no repeated edges or vertices. An element of a graph is an edge or a vertex of the graph. The length of a vertex-path (resp. an edge-path, a mixed-path) is the number of vertices of the path (resp. the number of edges, the number of edges and vertices). In other words, the length is the number of elements.

We color graphs element by element, but when we color an edge it might be the case that one or both of its vertices are not colored yet. Thus the graph restricted to the colored elements is not necessarily a proper graph in the sense that some edges might be missing one or two vertices. But for our inductive approach to hold, we need our result to hold for such objects. We do not want to formalize this notion, but whenever we remove a vertex from a graph all its adjacent edges remain in the graph and are still adjacent to one another. Thus, if $S$ is a set of edges or vertices of a graph $G$, we denote by $G-S$ the graph obtained by deleting exactly the vertices and edges of $S$ (that is, we do not remove edges connected to some vertices of $S$ unless they also belong to $S$ ). Also if two edges are connected by a vertex $v$ then they are still considered to be connected in $G-v$ and in particular they can still appear consecutively in an edge-path of $G-v$ (similarly two adjacent vertices are still adjacent even if we remove their shared edge and they can still be consecutive in a vertex-path). We use the same notation to remove a vertex-path, an edge-path or a mixed-path. For instance, if $p=\left(v_{1}, v_{2}, \ldots, v_{n}\right)$ is a vertex-path of $G$, then $G-p$ does not contain the vertixes $v_{1}, v_{2}, \ldots$ but it still contains the edge $\left\{v_{1}, v_{2}\right\}$.

\subsection{Weak total Thue coloring}

Let us recall that a coloring of the edges and the vertices of a graph is a weak total Thue coloring if the sequence of colors of any mixed-path is non-repetitive.

Theorem 5. For every graph $G$ with maximum degree $\Delta \geqslant 1$,

$$
\pi_{\mathrm{Twch}}(G) \leqslant 6 \Delta \text {. }
$$

Given a graph $G$, a set $S$ of edges and vertices of $G$ and a list assignment $L$ of $G$, the set $C_{L}(G-S)$ is the set of weak total Thue $L$-colorings of $G-S$.

Lemma 6. Let $\Delta \geqslant 2$ be an integer. Let $G$ be a graph of maximal degree at most $\Delta$ and $L$ be a list assignment of $G$. Suppose each list is of size at least $6 \Delta$ then for any vertex or edge $x$ of $G$ :

$$
\left|C_{L}(G)\right| \geqslant 3 \Delta\left|C_{L}(G-x)\right|
$$

Proof. Let us show this by induction on the sum of the number of vertices and edges of $G$. This is true for the graph with a single vertex since the empty graph admits exactly one coloring. Let $n$ be an integer such that the Lemma holds for any graph with less than $n$ vertices and edges.

Let $G=(V, E)$ be a graph with $|E|+|V|=n$ of maximal degree at most $\Delta$ and $L$ be a list assignment of $G$ such that each list is of size at least $6 \Delta$. Let $x$ be an edge or a vertex of $G$. 
Let $F$ be the set of $L$-colorings of $G$ that are not weak total Thue colorings but whose restriction to $G-x$ is a weak total Thue coloring. Then

$$
\left|C_{L}(G)\right|=6 \Delta\left|C_{L}(G-x)\right|-|F|
$$

In order to upper-bound the size of $F$, let $F_{i}$ be the set of colorings from $F$ that contains a mixed-path of length $2 i$ whose sequence of colors is a square ${ }^{2}$. Clearly $|F| \leqslant \sum_{i \geqslant 1}\left|F_{i}\right|$. For any coloring $c$ from $F_{i}$ there is a mixed-path $p$ of length $2 i$ such that

- the sequence of colors of $p$ is a square,

- $p$ contains $x$ and let us call $p^{\prime}$ the half of $p$ that contains $x$,

- the restriction of $c$ to $G-p^{\prime}$ is non-repetitive,

- $p$ and the restriction of $c$ to $G-p^{\prime}$ uniquely determines $c$ (since the second half of the square is identical to the first half).

Given $p$ and $p^{\prime}$ the number of such colorings in $F_{i}$ is at most $\left|C_{L}\left(G-p^{\prime}\right)\right|$, but since $p^{\prime}$ contains $x$ and $n-1$ other elements our induction hypothesis implies

$$
\left|C_{L}\left(G-p^{\prime}\right)\right| \leqslant \frac{\left|C_{L}(G-x)\right|}{(3 \Delta)^{i-1}}
$$

If $x$ is a vertex then there are at most $i \Delta^{i}$ mixed-paths of length $2 i$ going through $x$. If $x$ is an edge then there are at most $2 i \Delta^{i-1} \leqslant i \Delta^{i}$ mixed-paths of length $2 i$ going through $x$. We deduce

$$
\left|F_{i}\right| \leqslant i \Delta^{i} \frac{\left|C_{L}(G-x)\right|}{(3 \Delta)^{i-1}}=i \Delta \frac{\left|C_{L}(G-x)\right|}{3^{i-1}} .
$$

Thus we can finally upper-bound $|F|$

$$
|F| \leqslant \Delta\left|C_{L}(G-x)\right| \sum_{i \geqslant 1} \frac{i}{3^{i-1}} \leqslant \frac{9}{4} \Delta\left|C_{L}(G-x)\right| .
$$

Together with equation (6), it implies

$$
\begin{aligned}
\left|C_{L}(G)\right| & \geqslant 6 \Delta\left|C_{L}(G-x)\right|-\frac{9}{4} \Delta\left|C_{L}(G-x)\right| \\
& \geqslant \frac{15}{4} \Delta\left|C_{L}(G-x)\right| \geqslant 3 \Delta\left|C_{L}(G-x)\right|
\end{aligned}
$$

which concludes our proof.

A better analysis leads to a leading coefficient $\gamma=5.21914$ instead of 6 ( $\gamma$ is a root of the polynomial $\left.-3-4 x-20 x^{2}+4 x^{3}\right)$. The same result can be shown with a longer proof relying on entropy-compression.

\footnotetext{
${ }^{2}$ Recall, that a mixed-path of length $2 i$ has $i$ edges and $i$ vertices.
} 


\subsection{Weak total Thue coloring for large maximal degree}

It is clear in the previous proof that the worst case is when $x$ is a vertex since a vertex belongs to at most $i \Delta^{i}$ mixed-paths of length $2 i$ while an edge belongs an to at most $2 i \Delta^{i-1}$ such mixed-paths. In fact, we can use two different "growth rates" to distinguish between these cases and improve our bound on the weak total Thue number of graphs for large values of $\Delta$. It is not clear whether this second bound can be proven by the entropy-compression method.

Theorem 7. For every graph $G$ with maximum degree $\Delta \geqslant 300$,

$$
\pi_{\mathrm{Twch}}(G) \leqslant\lceil 4.25 \Delta\rceil .
$$

This is a simple corollary of the following Lemma.

Lemma 8. Let $\Delta \geqslant 300$ be an integer. Let $G$ be a graph of maximal degree at most $\Delta$ and $L$ be a list assignment of $G$. Suppose each list is of size at least $4.25 \Delta$ then for any edge e of $G$,

$$
\left|C_{L}(G)\right| \geqslant 4.2 \Delta\left|C_{L}(G-e)\right|
$$

and for any vertex $v$ of $G$,

$$
\left|C_{L}(G)\right| \geqslant 1.62 \Delta\left|C_{L}(G-v)\right|
$$

Proof. The proof is similar to the proof of Lemma 6. The induction is the same and we keep the notations $F, F_{i}$ and $p^{\prime}$, but we distinguish between edges and vertices to obtain better upper-bounds on the size of $F_{i}$.

Let us start with the proof of equation (7) when $e$ is an edge. There are at most $2 i \Delta^{i-1}$ mixed-paths of length $2 i$ going through $e$ and the induction hypothesis implies that $\left|C_{L}(G)\right| \geqslant 1.62 \Delta\left|C_{L}(G-x)\right|$, for any element $x$ of $G$. Thus we can use the exact same argument as in the previous proof to obtain

$$
\begin{aligned}
\left|C_{L}(G)\right| & \geqslant 4.25 \Delta\left|C_{L}(G-e)\right|-\sum_{i \geqslant 1} \frac{2 i \Delta^{i-1}\left|C_{L}(G-e)\right|}{(1.62 \Delta)^{i-1}} \\
& \geqslant\left|C_{L}(G-e)\right| \Delta\left(4.25-\frac{2}{\Delta} \sum_{i \geqslant 1} \frac{i}{1.62^{i-1}}\right) \\
& \geqslant\left|C_{L}(G-e)\right| \Delta\left(4.25-\frac{2}{\Delta} \frac{1.62^{2}}{0.62^{2}}\right) .
\end{aligned}
$$

Since $\Delta>300$, numerical computations yield

$$
\left|C_{L}(G)\right| \geqslant 4.2 \Delta\left|C_{L}(G-e)\right| .
$$

which concludes the proof of equation (7). 
We now prove equation (8) when $v$ is a vertex. The half $p^{\prime}$ of a mixed-path of length $2 i$ containing $v$ contains at least $\left\lceil\frac{i-1}{2}\right\rceil$ edges. Thus in this case our induction hypothesis implies

$$
\left|C_{L}\left(G-p^{\prime}\right)\right| \leqslant \frac{\left|C_{L}(G-v)\right|}{\left(\sqrt{1.62 \times 4.2 \Delta)^{i-1}} .\right.}
$$

Moreover, there are at most $i \Delta^{i}$ mixed-paths of length $2 i$ going through $v$ which gives:

$$
\begin{aligned}
\left|C_{L}(G)\right| & \geqslant 4.25 \Delta\left|C_{L}(G-v)\right|-\sum_{i \geqslant 1} \frac{i \Delta^{i}\left|C_{L}(G-v)\right|}{(\sqrt{1.62 \times 4.2} \Delta)^{i-1}} \\
& \geqslant\left|C_{L}(G-v)\right| \Delta\left(4.25-\sum_{i \geqslant 1} \frac{i}{(\sqrt{1.62 \times 4.2})^{i-1}}\right) .
\end{aligned}
$$

Numerical computations give

$$
\left|C_{L}(G)\right| \geqslant 1.62 \Delta\left|C_{L}(G-v)\right|
$$

which conclude our proof.

\subsection{Total Thue coloring}

Let us recall that a coloring of the edges and the vertices of a graph is a total Thue coloring if the sequence of colors of any mixed-path, any vertex-path and any edge-path is non-repetitive.

Theorem 9. For every graph $G$ with maximum degree $\Delta$,

$$
\pi_{\mathrm{Tch}}(G) \leqslant \Delta^{2}+\frac{3}{2^{1 / 3}} \Delta^{5 / 3}+8 \Delta^{4 / 3}+1 .
$$

This is a simple Corollary of Lemma 10. For any set $S$ of edges and vertices of a graph $G$ and any list assignment of $G$, the set $C_{L}(G-S)$ is the set of total Thue $L$-colorings of $G-S$.

Lemma 10. Let $\Delta \geqslant 2$ be an integer and $\gamma=\frac{3}{2^{1 / 3}}+8 \Delta^{-1 / 3}$. Let $G$ be a graph of maximal degree at most $\Delta$ and $L$ be a list assignment of $G$. Suppose each list is of size at least $\Delta^{2}\left(1+\gamma \Delta^{-1 / 3}\right)$ then for any vertex or edge $x$ of $G$ :

$$
\left|C_{L}(G)\right| \geqslant \Delta^{2}\left(1+2^{2 / 3} \Delta^{-1 / 3}\right)\left|C_{L}(G-x)\right| .
$$

Proof. Let us proceed by induction on the sum of the number of vertices and edges of $G$. This is true for the graph with a single vertex since the empty graph admits exactly one coloring. Let $n$ be an integer such that the Lemma holds for any graph with less than $n$ elements (vertices and edges).

Let $G=(V, E)$ be a graph with $|E|+|V|=n$ of maximal degree at most $\Delta$ and $L$ be a list assignment of $G$ such that each list is of size at least $\Delta^{2}\left(1+\gamma \Delta^{-1 / 3}\right)$. Let $x$ be an edge or a vertex of $G$. 
Let $F$ be the set of $L$-colorings of $G$ that are not total Thue coloring but whose restriction to $G-x$ is a total Thue coloring. Then

$$
\left|C_{L}(G)\right|=\Delta^{2}\left(1+\gamma \Delta^{-1 / 3}\right)\left|C_{L}(G-x)\right|-|F| .
$$

We need to upper-bound the size of $F$. Let $M_{i}$ be the set of colorings from $F$ that contains a mixed-path of length $2 i$ whose sequence of colors is repetitive. If $x$ is an edge (resp. a vertex) let $S_{i}$ be the set of colorings from $F$ that contain an edge-path (resp. a vertexpath) of length $2 i$ whose sequence of colors is repetitive. Clearly $|F| \leqslant \sum_{i \geqslant 1}\left(\left|M_{i}\right|+\left|S_{i}\right|\right)$.

For any coloring $c$ from $M_{i}$ there is mixed-path $p$ of length $2 i$ such that

- the sequence of colors of $p$ is a square,

- $p$ contains $x$ and let us call $p^{\prime}$ the half of $p$ that contains $x$,

- the restriction of $c$ to $G-p^{\prime}$ is non-repetitive,

- $p$ and the restriction of $c$ to $G-p^{\prime}$ uniquely determines $c$ (since the second half of the square is identical to the first half).

Given $p$ and $p^{\prime}$ the number of such colorings from $M_{i}$ is at most $\left|C_{L}\left(G-p^{\prime}\right)\right|$, but since $p^{\prime}$ contains $x$ and $n-1$ other elements our induction hypothesis implies that this quantity is bounded by

$$
\left|C_{L}\left(G-p^{\prime}\right)\right| \leqslant \frac{\left|C_{L}(G-x)\right|}{\left(\Delta^{2}\left(1+2^{2 / 3} \Delta^{-1 / 3}\right)\right)^{i-1}} .
$$

If $x$ is a vertex then there are at most $i \Delta^{i}$ mixed-paths of length $2 i$ going through $x$. If $x$ is an edge then there are at most $2 i \Delta^{i-1} \leqslant i \Delta^{i}$ mixed-paths of length $2 i$ going through $x$. We deduce

$$
\left|M_{i}\right| \leqslant i \Delta^{i} \frac{\left|C_{L}(G-x)\right|}{\left(\Delta^{2}\left(1+2^{2 / 3} \Delta^{-1 / 3}\right)\right)^{i-1}}=i \Delta \frac{\left|C_{L}(G-x)\right|}{\left(\Delta\left(1+2^{2 / 3} \Delta^{-1 / 3}\right)\right)^{i-1}} .
$$

If the sequence of colors of an edge-path (resp. a vertex-path) is a square we can also recover the coloring of the full path by knowing only the first half of it. Moreover, there are at most $2 i \Delta^{2 i-1}$ edge-paths of length $2 i$ going through a given edge and at most $i \Delta^{2 i-1} \leqslant 2 i \Delta^{2 i-1}$ vertex-paths of length $2 i$ going through a given vertex. Using the same idea we can bound the size of $S_{i}$ by

$$
\left|S_{i}\right| \leqslant 2 i \Delta^{2 i-1} \frac{\left|C_{L}(G-x)\right|}{\left(\Delta^{2}\left(1+2^{2 / 3} \Delta^{-1 / 3}\right)\right)^{i-1}}=2 i \Delta \frac{\left|C_{L}(G-x)\right|}{\left(1+2^{2 / 3} \Delta^{-1 / 3}\right)^{i-1}} .
$$

We can now upper-bound $|F|$.

$$
\begin{aligned}
|F| & \leqslant \Delta\left|C_{L}(G-x)\right| \sum_{i \geqslant 1} \frac{2 i}{\left(1+2^{2 / 3} \Delta^{-1 / 3}\right)^{i-1}}+\frac{i}{\left(\Delta\left(1+2^{2 / 3} \Delta^{-1 / 3}\right)\right)^{i-1}} \\
& \leqslant \Delta\left|C_{L}(G-x)\right|\left(1+\left(2+\frac{1}{\Delta}\right) \sum_{i \geqslant 1} \frac{i}{\left(1+2^{2 / 3} \Delta^{-1 / 3}\right)^{i-1}}\right)
\end{aligned}
$$




$$
\begin{aligned}
& \leqslant \Delta\left|C_{L}(G-x)\right|\left(1+\left(2+\frac{1}{\Delta}\right) \frac{\left(1+2^{2 / 3} \Delta^{-1 / 3}\right)^{2}}{\left(2^{2 / 3} \Delta^{-1 / 3}\right)^{2}}\right) \\
& \leqslant \Delta^{5 / 3}\left|C_{L}(G-x)\right|\left(\Delta^{-2 / 3}+\left(2+\frac{1}{\Delta}\right)\left(2^{-2 / 3}+\Delta^{-1 / 3}\right)^{2}\right)
\end{aligned}
$$

Since $\Delta>1\left(\right.$ and $\left.\Delta^{-1 / 3}>\Delta^{-2 / 3}\right)$ we finally get

$$
|F| \leqslant \Delta^{5 / 3}\left|C_{L}(G-x)\right|\left(2^{-1 / 3}+8 \Delta^{-1 / 3}\right) \text {. }
$$

Together with equation (9), it implies

$$
\begin{aligned}
\left|C_{L}(G)\right| & \geqslant \Delta^{2}\left(1+\gamma \Delta^{-1 / 3}\right)\left|C_{L}(G-x)\right|-\Delta^{5 / 3}\left|C_{L}(G-x)\right|\left(2^{-1 / 3}+8 \Delta^{-1 / 3}\right) \\
& \geqslant \Delta^{2}\left|C_{L}(G-x)\right|\left(1+\Delta^{-1 / 3}\left(\gamma-\left(2^{-1 / 3}+8 \Delta^{-1 / 3}\right)\right)\right) .
\end{aligned}
$$

Substituting $\gamma=\frac{3}{2^{1 / 3}}+8 \Delta^{-1 / 3}$, we finally get

$$
\left|C_{L}(G)\right| \geqslant \Delta^{2}\left(1+2^{2 / 3} \Delta^{-1 / 3}\right)\left|C_{L}(G-v)\right|
$$

which concludes this proof.

This upper-bound also holds for non-repetitive vertex coloring and non-repetitive edge coloring which provides the following Corollary.

Corollary 11. For every graph $G$ with maximum degree $\Delta$,

$$
\pi_{\mathrm{ch}}^{\prime}(G) \leqslant \Delta^{2}+\frac{3}{2^{1 / 3}} \Delta^{5 / 3}+8 \Delta^{4 / 3}+1 .
$$

This bound is better than the upper-bound $\pi_{l}^{\prime}(G) \leqslant \Delta^{2}+2^{4 / 3} \Delta^{5 / 3}+O\left(\Delta^{4 / 3}\right)$ given in $[10]\left(2^{4 / 3} \approx 2.52 \ldots\right.$ and $\left.\frac{3}{2^{1 / 3}} \approx 2.38 \ldots\right)$. One can also easily improve the coefficient of $\Delta^{4 / 3}$ in our bound with a more careful analysis (at least as low as $1+2^{4 / 3}$ in the case of total Thue coloring and $2^{4 / 3}$ in the case of non-repetitive edge coloring).

\section{Conclusion}

Most results in this paper can be obtained with entropy-compression. However, our approach is much simpler to use and provides exponential lower bounds on the number of satisfying assignments. Moreover, it is not clear that Theorem 7 can be proven by entropy-compression. Our approach can obviously be generalized outside of the scope of non-repetitive coloring and we can for instance provide simpler proofs of all the result from $[9]$.

Our proof technique is also strongly related to the Lovász Local Lemma. We show lemmas of the form "with $\gamma$ colors, coloring a new vertex multiply the number of valid colorings by $\alpha$ " which is equivalent to "when adding a new vertex the probability for a random coloring to be valid is multiplied by at least $\frac{\alpha}{\gamma}$ " (remark that $\frac{\alpha}{\gamma}$ is a quantity smaller than 1). Proofs of the Lovász Local Lemma are based on idea similar to the second version of this statement. In fact, some SAT versions of the Lovász Local Lemma are also easy to obtain with our approach, but once again we get the same bounds. 


\section{Acknowledgment}

I wish to thank Gwenaël Joret and William Lochet for comments on earlier drafts. I also wish to thank Lucile without whom the covid-19 lockdown would have been much more unpleasant and less productive.

\section{References}

[1] N. Alon, J. Grytczuk, M. Haluszcza, and O. Riordan. Nonrepetitive colorings of graphs. Random Structures \& Algorithms, 21:336-346, 2002.

[2] J. P. Bell and T. L. Goh. Exponential lower bounds for the number of words of uniform length avoiding a pattern. Information and Computation, 205(9):1295-1306, 2007.

[3] A. Bernshteyn. The local cut lemma. European Journal of Combinatorics, 63:95-114, 2017.

[4] J. Berstel. Axel Thue's papers on repetitions in words: a translation. Publications du LaCIM 20, Université du Québec à Montréall, 1995.

[5] F. Blanchet-Sadri and B. Woodhouse. Strict bounds for pattern avoidance. Theoretical Computer Science, 506:17-28, 2013.

[6] S. Czerwiński and J. Grytczuk. Nonrepetitive colorings of graphs. Electronic Notes in Discrete Mathematics, 28:453-459, 2007. 6th Czech-Slovak International Symposium on Combinatorics, Graph Theory, Algorithms and Applications.

[7] V. Dujmović, L. Esperet, G. Joret, B. Walczak, and D.R. Wood. Planar graphs have bounded nonrepetitive chromatic number. Advances in Combinatorics, 2020:5.

[8] V. Dujmović, G. Joret, J. Kozik, and D. R. Wood. Nonrepetitive colouring via entropy compression. Combinatorica, 36(6):661-686, Dec 2016.

[9] L. Esperet and A. Parreau. Acyclic edge-coloring using entropy compression. European Journal of Combinatorics, 34(6):1019-1027, 2013.

[10] D. Gonçalves, M. Montassier, and A. Pinlou. Entropy compression method applied to graph colorings. arXiv:1406.4380, 2014.

[11] J. Grytczuk, J. Kozik, and P. Micek. New approach to nonrepetitive sequences. Random Struct. Algorithms, 42(2):214-225, March 2013.

[12] J. Grytczuk, J. Przybyło, and X. Zhu. Nonrepetitive list colourings of paths. Random Structures \& Algorithms, 38(1-2):162-173, 2011.

[13] J. Harant and S. Jendrol. Nonrepetitive vertex colorings of graphs. Discrete Mathematics, 312(2):374-380, 2012.

[14] R. A. Moser and G. Tardos. A Constructive Proof of the General Lovász Local Lemma. J. ACM, 57(2), February 2010.

[15] P. Ochem. Doubled patterns are 3-avoidable. Electronic Journal of Combinatorics, 23(1), 2016. 
[16] N. Rampersad. Further applications of a power series method for pattern avoidance. Electronic Journal of Combinatorics, 18:134, 2011.

[17] J. Schreyer and E. Škrabuvláková. Total Thue colourings of graphs. European Journal of Mathematics, (1):186-197, 2015.

[18] A. Thue. Über unendliche Zeichenreihen. 'Norske Vid. Selsk. Skr. I. Mat. Nat. Kl. Christiania, 7:1-22, 1906.

[19] A. Thue. Über die gegenseitige Lage gleicher Teile gewisser Zeichenreihen. Norske Vid. Selsk. Skr. I. Mat. Nat. Kl. Christiania,, 10:1-67, 1912.

[20] E. Škrabuláková. The Thue choice number versus the Thue chromatic number of graphs. arXiv:1508.02559, August 2015. 